\title{
PERCEPCIONES, CONOCIMIENTO LOCAL Y EXPECTATIVAS DE CAMPESINOS CAFETALEROS EN PURISCAL, COSTA RICA
}

\author{
Jairo Mora-Delgado ${ }^{1}$ \\ Felipe Montoya-Greenheck ${ }^{2}$ \\ Carlos Ramírez-Martínez ${ }^{3}$ \\ jrmora@ut.edu.co
}

Manizales, 2009-10-06 (Rev. 2009-11-06)

\begin{abstract}
RESUMEN
El propósito de este estudio fue sistematizar las percepciones y saberes locales emitidos por una comunidad campesina de Costa Rica, principalmente en cuanto a su visión del estado actual y las expectativas para el futuro de la actividad cafetera. Se realizaron entrevistas en profundidad para indagar la percepción sobre el estado actual de la caficultura y las expectativas para el futuro. Las entrevistas se desarrollaron en 39 fincas cafetaleras del cantón de Puriscal, Costa Rica. El estudio tomó elementos de los procedimientos y técnicas de enfoques cualitativos como grounded theory y sistematización de experiencias. Los temas abordados fueron enfocados hacia tres tópicos: estado actual y futuro de la actividad cafetera; opiniones sobre la caficultura orgánica, y expectativas y necesidades de capacitación. A pesar de que el mayor número de enunciados sugiere una actitud pesimista frente a la crisis de esta actividad, casi la mitad de ellos denotan una actitud optimista frente a esta actividad. El mayor número de enunciados sistematizados evidencia una actitud optimista frente a la producción orgánica. La mayor parte de expectativas denotan la necesidad de capacitarse en manejo de abonos orgánicos y control de enfermedades.
\end{abstract}

\section{PALABRAS CLAVE}

Análisis de texto, caficultura orgánica, enunciados unitarios, investigación cualitativa.

\section{PERCEPTIONS, LOCAL KNOWLEDGE AND EXPECTATIONS OF PEASANT COFFEE FARMERS IN PURISCAL, COSTA RICA}

\begin{abstract}
This study aims to systematization ofcapture the experience of both perceptions and local knowledge of a Peasant community from Costa Rica, mainly, about his on their vision and expectation on the coffee growingth activity. Interview iln- depth interviews were done carried out to inquiry perceptions on current state of coffee production systems and expectations at regarding future of coffee productiongrowers. Interviews were applieds in 39 coffee farms of Cantón of Puriscal, Costa Rica. This study used methods and techniques from qualitative approaches such as likethe grounded theory and the systematization of experiences. The issues treated were related with to three topics: present and future state of coffee production systems; opinions on organic coffee production systems, and training expectations and requirements of training. Even though the most statements suggested a pessimistic attitude in frontregarding the crisis of coffee productionactivity, almostready half of producers noted an optimistic attitude towardsin front of the future. The mMost of the systemized statements evidenced an optimistic attitude regardingin front organic coffee production system. The mostmajority of expectations systematized indicate the need requirements of training in about both management of organic fertilizers management and disease control of diseases.
\end{abstract}

KEY WORDS: Text analysis, organic coffee production, unit statements, qualitative inquiryresearch. 


\section{INTRODUCCIÓN}

El presente artículo aborda una comunidad campesina productora de café de la Zona Central de Costa Rica. La categoría social denominada "campesino" con sus percepciones, saberes y sus sistemas de producción, constituyen un grupo social importante en un mundo globalizado. En especial, el conocimiento desarrollado en estas comunidades cada día cobra más importancia.

El conocimiento local de las sociedades campesinas e indígenas en países en desarrollo es extenso, sistemático y utilizado, en muchas ocasiones, para tomar importantes decisiones de manejo agrícola. Este tipo de saber popular es uno de los factores que influyen en los procesos de decisión del agricultor, aunque también existen factores y limitantes exógenos que pueden desempeñar un papel fundamental en la conducta y actitud del productor (Sain 1999).

El conocimiento local es el adquirido por un grupo de personas y definido por la zona o localidad donde ellas habitan (Sinclair et al. 1993, Sinclair et al. 1999). Los estudios de conocimiento local distinguen entre tres grandes categorías: percepciones, taxonomías y modelos causales. Las percepciones pueden ser ampliamente compartidas o particulares a un individuo y sin ninguna o poca consistencia entre individuos y pueden ser idiosincrásicas, como por ejemplo la realización de prácticas agrícolas en función de las fases de la luna (Sain 1999) u otras creencias. Cuando de las percepciones se abstraen categorías con nombres y propiedades definidas se las sistematiza, relacionándolas entre sí de manera jerárquica, generando taxonomías. Gould y Vrba (1982), citando un texto de Michel Foucault, sugieren que cuando se conoce por qué y cómo clasifica la gente, en cierta forma se entiende cómo piensa. En su opinión, las taxonomías no son categorías neutrales o arbitrarias para un conjunto de conceptos inmutables, más bien reflejan (o incluso crean) diferentes teorías acerca de la estructura del mundo.

Este estudio se hizo con el propósito de sistematizar las percepciones y saberes locales emitidos por una comunidad de productores en una zona de economía campesina de Costa Rica, principalmente, en cuanto a su visión del estado actual y las expectativas para el futuro. El trabajo constituye una aproximación desde una perspectiva emic, desarrollado con base en entrevistas individuales y en talleres grupales sobre la caficultura.

\section{METODOLOGÍA}

El tema de análisis de este artículo constituye una parte de un proceso de investigación de la tesis doctoral del primer autor, cuyo trabajo de campo comenzó en el año 2000 y se prolongó hasta el 2003, pero sólo hasta agosto de 2004 se culminó el ordenamiento y posterior análisis de la base de datos, que dio origen al presente artículo.

Siguiendo un camino inductivo para la construcción de la teoría, el estudio tomó elementos de los procedimientos y técnicas del enfoque que en inglés se conoce como grounded theory (Strauss y Corbin 1990) y en los trabajos de Jara (1998) sobre sistematización de experiencias. Los temas abordados fueron enfocados hacia tres tópicos: (a) estado actual y futuro de la caficultura; (b) opiniones sobre la caficultura orgánica, y (c) expectativas y necesidades de capacitación. Dichas entrevistas fueron grabadas y posteriormente transcritas; los textos fueron desagregados en enunciados unitarios, siguiendo la metodología propuesta en el programa Agroecological Knowledge Toolkit, AKT5 (Dixon et al. 2001).

Los enunciados unitarios contienen conocimiento comprensible sin referencia a otra expresión y no pueden ser desagregados en otros enunciados (Dixon et al. 2001); son la unidad textual mínima con sentido. Los enunciados unitarios se dividen en dos categorías: enunciados de atributo-valor y enunciados binarios. Los primeros describen mediante atributos y califican mediante valores cualitativos o cuantitativos; un valor siempre está asociado a un atributo. Los segundos establecen relaciones entre dos entidades, que pueden ser de cuatro tipos: causalidad, comparación, condición o, simplemente, declaraciones de enlace. Se estableció un tipo en esta categoría que no está contemplado claramente en la metodología de Dixon et al. (2001), y que aquí se denomina enunciados propositivos; estos dan idea del "qué hacer" o proponen acciones explícitamente. Estas categorías de enunciados fueron cruzadas en una matriz con cuatro categorías de valoración cualitativa, en función del tenor de la actitud que denotara el hablante. Las cuatro categorías de actitud fueron: optimismo (O), pesimismo (P), incertidumbre (I) y neutralidad (N). 
Se realizaron entrevistas en profundidad para indagar la percepción general sobre la caficultura, el estado actual de la actividad y las expectativas para el futuro. Las entrevistas se desarrollaron en diferentes escenarios (casa del productor, salas de reunión o caminatas por los predios) de 39 fincas cafetaleras del cantón de Puriscal, región central de Costa Rica. El Cantón de Puriscal limita al norte con los Cantones de San José y Mora, Turrubares por el occidente, Acosta por el oriente y Parrita por el Sur.

Las fincas cafetaleras estudiadas están distribuidas en las localidades de Candelarita, Bajo la Legua, Cerbatana, Cañales Abajo, Junquillo, Túfares, La Fila, Santa Marta, Grifo Alto, Barrio Mora, Santa Bárbara y La Legua aproximadamente entre los paralelos $9^{\circ} 45^{\prime}$ y $9^{\circ} 54^{\prime} \mathrm{N}$ y los meridianos $84^{\circ} 17^{\prime}$ y $84^{\circ} 25^{\prime} \mathrm{O}$. La precipitación media anual es de alrededor de $2050 \mathrm{~mm}$, con una estación seca pronunciada que va desde diciembre hasta abril. La temperatura media anual varía entre 19,6 y 22,9 oC, y la zona de vida es Bosque Muy Húmedo Premontano (Holdridge 1978).

\section{RESULTADOS Y DISCUSIÓN}

En el fluir del discurso de los entrevistados emergen distintos temas. Muchas veces se expresan en palabras clave ${ }^{4}$, en torno a las cuales se van configurando variados campos semánticos. Es decir, a partir de un sentido de base al cual remiten las palabras, en cada entrevistado se genera una red de asociaciones que transforman y delimitan un campo de sentido. En otras palabras, los campos semánticos se constituyen de acuerdo con un sistema de sentidos cuya delimitación es configurada por el contexto del hablante y de la situación de la entrevista.

El corte transversal de las entrevistas permitió observar una diversidad de énfasis en cuanto a los temas abordados por los hablantes. Cada entrevistado eligió de qué hablar — dentro del universo del momento situacional de la caficultura- y, por lo tanto, desarrolló su discurso en torno a distintos aspectos de la dinámica actual de esta actividad económica. La percepción se expresa en opiniones y, en el sentido más restringido, el término percepción se distingue de la sensación en cuanto indica un proceso cognoscitivo de unificación de una multiplicidad de sensaciones (Firenze, 2003).

Las opiniones generalmente se desagregan en enunciados unitarios y se clasifican en función de su grado de complejidad. Aparentemente, los enunciados son innumerables, sin embargo, al desagregar las opiniones en enunciados unitarios se encontró que muchos de ellos tenían diferente estructura pero el mismo sentido, o eran expresadas con palabras diferentes, o con las mismas palabras pero en distinto orden. Una vez desagregados los textos en enunciados unitarios, se construyeron 48 enunciados relacionados con la percepción de la caficultura en general y 33 opiniones sobre la caficultura orgánica. Estos enunciados se clasificaron en las seis categorías de enunciados y cuatro actitudes explicadas en la metodología.

\section{a. Acerca de la caficultura: su estado actual y su futuro.}

Los enunciados declarativos o de enlace constituyen relaciones que dan una idea de percepciones simples, y muchas veces denotan enunciados informativos neutrales, sentencias, o dan idea de la actitud del productor frente a la situación de la caficultura. Al respecto Pérez (2009) manifiesta que el conocimiento declarativo consiste en información sobre cómo está organizado el mundo y lo que sucede en él, así, la "memoria declarativa", que conserva este conocimiento, es una memoria semántica compuesta de numerosos "nodos" -unidades cognitivas- formando una red.

El $19 \%$ de los enunciados son de este tipo y los informantes principalmente fueron caficultores convencionales. Si bien el sentido de estos enunciados es neutral, las emociones manifestadas por los hablantes denotan conformidad o que el productor posiblemente haya perdido interés en el asunto ante las repetidas crisis. En contraste, otras opiniones -enunciados de atributo-valor- expresan algún tipo de emoción o actitud frente a un hecho, lo cual demanda un mayor nivel analítico, ya que la valoración per se podría tomarse como una aproximación a la explicación. En estos enunciados, el productor emite calificativos sobre la actividad cafetalera, lo cual en cierta forma explica su actitud. El $16 \%$ de los enunciados fueron de este tipo; las opiniones mencionan principalmente los precios bajos e impredecibles, la incertidumbre del mercado y los altos costos de producción. 
Los enunciados causales y comparativos constituyen una forma de modelos mentales ya que explicitan las relaciones causales entre los diferentes componentes del sistema (Johnson-Laird, citado por Pérez 2009), brindan razones por las cuales los productores adoptan actitudes de aceptación o rechazo de una práctica o modelo. El $19 \%$ de los enunciados correspondieron a relaciones de causalidad, es decir, aquellos que intentan adjudicar una causa a la crisis de la caficultura. Sobresalen los argumentos causales de la crisis atribuidas a los bajos precios, aunque también se mencionan los altos costos de producción y la superproducción en el ámbito global. El común denominador de estos enunciados de causalidad es que tratan de identificar un responsable de la crisis. Por el contrario, otros enunciados causales tratan de justificar la decisión de mantener el cultivo a pesar de la crisis: "Mantengo el café allí porque con eso me crié" (...) "ya que el café está ahí sembrado, ahí que se quede".

Los enunciados comparativos demuestran una mayor capacidad analítica del hablante, pues en ellos se establecen comparaciones entre conceptos. Sin embargo, este tipo de enunciados fueron los menos frecuentes $(6,3 \%)$. Las comparaciones reportadas se establecen entre costos e ingresos de la actividad cafetalera, aunque un informante estableció comparaciones de competitividad del cultivo en relación con las condiciones agroecológicas.

Los enunciados condicionales establecen una relación de la forma: "si está presente X, sucede Y" o "sucede $X$ aunque $Y$ ". Este tipo de enunciados representan el $19 \%$ del total y fueron emitidos principalmente en las entrevistas con caficultores orgánicos (12,5\% de los enunciados). Ellos expresaron su disposición a continuar practicando la caficultura orgánica a pesar de los bajos precios. Estos enunciados pueden constituir un intento de teoría, esto es una explicación de porqué se da la relación causa-efecto (Sain 1999).

Los enunciados propositivos denotan un mayor grado de actitud analítica del hablante, pues son el resultado de análisis previos. Estos enunciados no se limitan a informar, calificar o explicar, sino que llegan a proponer opciones y acciones. Este tipo de enunciados fue el más frecuente (22\%), en particular los que manifiestan las necesidades de intervenciones institucionales y estatales o los que proponen un margen de espera hasta que mejoren los precios del grano (Figura 1). Cada nueva oración o proposición agrega informaciones al modelo, así, para aprehender la compatibilidad de las informaciones y del modelo, puede ser necesario hacer uso simultáneamente de conocimientos generales y de conocimientos específicos no explícitos en el texto (Garnham y Oakhill, 1996).

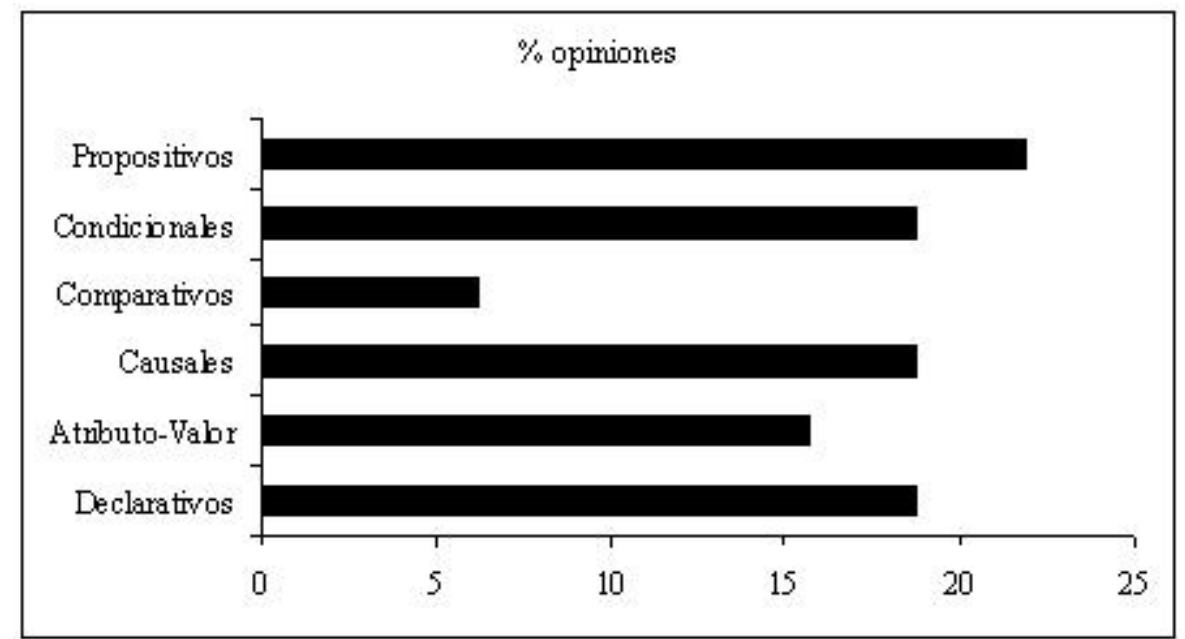

Figura 1. Percepciones de los productores respecto a la caficultura en Puriscal, Costa Rica. 


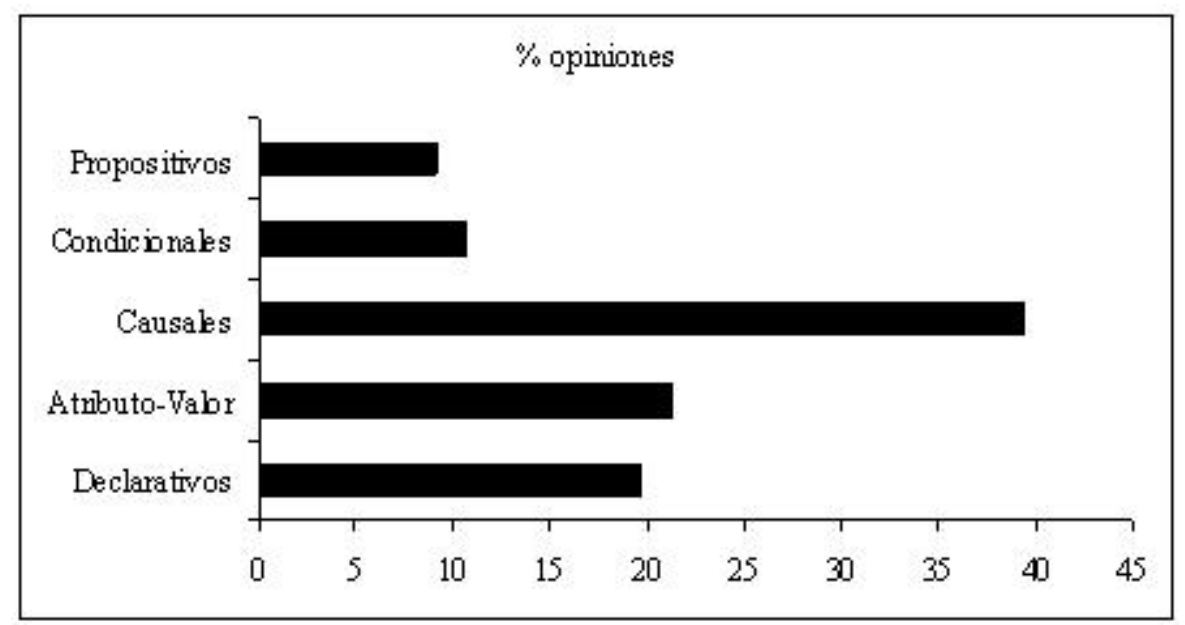

Figura 2. Percepciones de los productores respecto a la caficultura orgánica en Puriscal, Costa Rica.

Los datos de la matriz cruzada (Cuadro 1) indican que, a pesar de que el mayor número de enunciados sugiere una actitud pesimista frente a la crisis de la caficultura, casi la mitad de ellos denotan una actitud optimista frente a esta actividad. Además, hay una fracción de enunciados que indican neutralidad frente al futuro y un pequeño grupo que expresa incertidumbre.

Cuadro 1. Matriz cruzada entre el número de enunciados, clasificados según las categorías de percepciones y la actitud frente al futuro de la caficultura en Puriscal, Costa Rica.

\begin{tabular}{|l|c|c|c|c|c|}
\hline \multirow{2}{*}{ Categoria de percepción } & \multicolumn{5}{|c|}{ Actitud } \\
\cline { 2 - 6 } & 0 & $\mathrm{P}$ & $\mathrm{I}$ & $\mathrm{N}$ & Total \\
\hline Declarativos & 2 & 6 & 0 & 4 & 12 \\
\hline Atributo valor & 0 & 4 & 6 & 0 & 10 \\
\hline Causal & 0 & 9 & 0 & 3 & 12 \\
\hline Comparativo & 0 & 3 & 0 & 1 & 4 \\
\hline Condicional & 9 & 3 & 0 & 0 & 12 \\
\hline Propositivo & 13 & 1 & 0 & 0 & 14 \\
\hline Total de enunciados & 24 & 26 & 6 & 8 & 64 \\
\hline
\end{tabular}

Optimismo (O), pesimismo (P), incertidumbre (I) Neutralidad (N).

\section{b. Acerca de la caficultura orgánica}

El análisis de texto de opiniones relacionadas con la caficultura orgánica, tema recurrente en los diálogos exploratorios, sugiere que los enunciados de atributo-valor y causales son los más comunes entre los informantes $(21,2 \%$ y $39,4 \%$, respectivamente).

Los enunciados causales son de la forma "X para Y" o "X causa Y". En esta categoría, sobresalen los enunciados que relacionan caficultura orgánica con conservación de los recursos naturales y la salud humana. Algunos ejemplos de dichos enunciados fueron "practico la caficultura orgánica para eliminar la contaminación del ambiente" y "practico agricultura orgánica para cuidar la salud de la familia". Asimismo, se esgrimieron razones de convicción para justificar la persistencia en la caficultura orgánica, 
principalmente por los caficultores afiliados a ANACOP. Los enunciados condicionales supeditan una decisión de cambio a una condición que limita la acción; dicha condición puede constituir una aversión al riesgo ("es difícil y riesgoso hacer la conversión tecnológica"; "no hay suficientes evidencias de que esa tecnología sea buena") o por una limitación aparentemente real del sistema (costos elevados, precios bajos, etc.). Los datos de la matriz cruzada (Cuadro 3 ) evidencian que un mayor número de enunciados sugiere una actitud optimista frente a la caficultura orgánica. Sólo 10 enunciados expresaron claramente una actitud pesimista y el resto desconocen los detalles de esta opción o denotan incertidumbre. Cabe mencionar que la mayoría de los enunciados optimistas provenían de productores orgánicos.

Cuadro 2. Matriz cruzada entre el número de enunciados clasificados según las categorías de percepciones y la actitud frente a la caficultura orgánica en Puriscal, Costa Rica.

\begin{tabular}{|l|c|c|c|c|c|}
\hline \multirow{2}{*}{\multicolumn{1}{c|}{}} & \multicolumn{5}{|c|}{ Actitud } \\
\cline { 2 - 6 } Categoria de percepción & 0 & $\mathrm{P}$ & $\mathrm{I}$ & $\mathrm{N}$ & Total \\
\hline Declarativos & 2 & 2 & 3 & 6 & 13 \\
\hline Atributo Valor & 9 & 3 & 2 & 0 & 14 \\
\hline Causal & 25 & 1 & 0 & 0 & 26 \\
\hline Condicional & 2 & 3 & 2 & 0 & 7 \\
\hline & 2 & 1 & 0 & 3 & 6 \\
\hline Propositivo & 40 & 10 & 7 & 9 & 66 \\
\hline Total de enunciados & & & & & \\
\hline
\end{tabular}

Optimismo $(\mathrm{O})$, pesimismo $(\mathrm{P})$, incertidumbre $(\mathrm{I})$ neutralidad $(\mathrm{N})$.

\section{c. Acerca de las dificultades en el ciclo productivo y las necesidades de capacitación}

Otro tópico planteado en los diálogos estaba orientado a conocer la percepción campesina con respecto a aspectos del ciclo productivo considerados como más problemáticos. Desde la percepción de los productores, estos aspectos constituyen puntos neurálgicos que hay que intervenir para coadyuvar a mejorar la productividad y enfrentar la crisis. El $30 \%$ de los productores consideraron la comercialización como el eslabón más problemático de la cadena productiva; el $25 \%$ identifica dificultades en el control de enfermedades; el 15\% en la etapa de semillero-almacigal y, el resto, se inclina por identificar problemas relacionados con la fertilización, control de malezas, cosecha y beneficio del grano.

Sin embargo, no hay concordancia entre la percepción sobre los aspectos más problemáticos del ciclo productivo y las necesidades de capacitación expresadas en las entrevistas. Por un lado, las mayores dificultades se asignan a aspectos de comercialización, mientras que las necesidades de capacitación expresadas por la mayoría de los productores apuntan al fortalecimiento de las prácticas de manejo, como el control de enfermedades y la elaboración y manejo de abonos orgánicos (Figura 3). Como consecuencia lógica, se esperaría que sus aspiraciones de capacitación sean una respuesta a los vacíos o dificultades expresadas como aspectos problemáticos, es decir, temas relacionados con la organización de productores, gestión de mercados alternativos, certificación, etc. Sin embargo, estos aspectos no fueron mencionados y sólo un $10,3 \%$ se refiere en términos generales a aspectos de beneficio y comercialización, principalmente por productor de café orgánico.

Las necesidades de capacitación en manejo de abonos orgánicos fueron expresadas por caficultores orgánicos y mixtos, en particular, los productores orgánicos afiliados a ANACOP consideran que ya han superado la etapa de aprender la fabricación y uso y ahora necesitan aprender a mejorar la calidad. Un $18 \%$ del total considera necesario capacitarse en todas las etapas del ciclo productivo. La necesidad de capacitación en todo el proceso y en manejo de plagas y enfermedades es más sentida por los caficultores TM y TC. Un importante porcentaje del total manifestó querer capacitarse en técnicas de 
beneficiado y comercialización del grano; en particular, los productores de café orgánico afiliados a ANACOP consideran necesaria esta capacitación puesto que ya han incursionado con cierto éxito en el beneficiado del café en finca e, inclusive, en el secado, molienda y tueste.

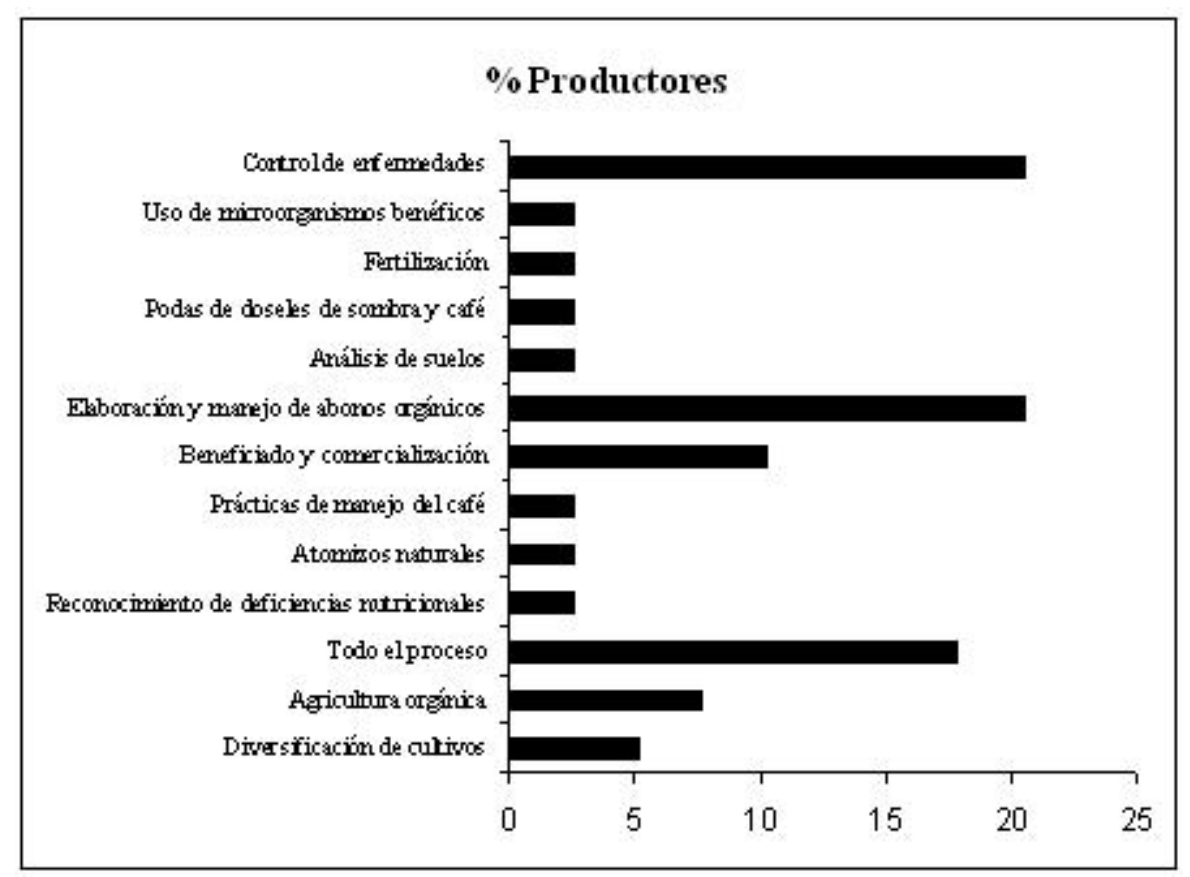

Figura 3. Necesidades de capacitación expresadas por los caficultores en Puriscal, Costa Rica.

\section{CONCLUSIÓN}

El conocimiento local se expresa en percepciones, decisiones y acciones. Las percepciones se legitiman en opiniones que varían dentro de un amplio rango de complejidad, que va desde los juicios de atributovalor hasta relaciones más complejas que proponen salidas o explican las condicionantes que afectan las decisiones.

Mejores condiciones de salud para las familias, el mejoramiento de las condiciones del suelo y la conservación de la biodiversidad son argumentos usados por los productores para tomar decisiones de cambio en la finca. Tales motivaciones están relacionadas con la expectativa de una mejora futura de la actividad cafetalera por su vínculo con externalidades como la generación de servicios ambientales o bienestar social. Estos argumentos son explícitos en los testimonios de los productores orgánicos, en tanto que los convencionales sólo los sugieren. En contraste, la cautela y la aversión al cambio que constituyen características de la cultura campesina posiblemente sean las principales razones que impulsan a los caficultores a tomar una actitud de espera a que mejoren las condiciones del mercado del café. 


\section{BIBLIOGRAFÍA}

- $\quad$ Dixon, HJ; Doores, JW; Joshi, L; Sinclair, FL. Agroforestry Knowlodge Toolkit for Windows for AKT5. Bangor, UK, University of Wales. 171 p. 2001. Garnham, A; Oakhill, J. Manual de Psicología del Pensamiento. PAIDÓS.

- Barcelona. Guiraud, P. La sémantique, "Que sais-je?". Paris, FR, PUF. 128 p. 1962.

- $\quad$ Firenze, A. El problema de la percepción y la fenomenología de Merleau-Ponty. O line. Sección Clínica de Barcelona - Instituto del Campo Freudiano. 2003. En linea: http://www.scb-icf.net/nodus/

- $\quad$ Gould, SJ; Vrba, ES. Exaptation-a missing term in the science of form. Paleobiology 8:4-15. 1982.

- Holdridge, LR. Ecología basada en zonas de vida. Instituto Interamericano Ciencias Agrícolas, San José. 216 p. 1978.

- Jara, O. Para sistematizar experiencias: una propuesta teórica y práctica . 3 ed. San José, CR, Alforja. 243 p. 1998.

- Mora-Delgado J. Tecnología, Conocimiento Local y Evaluación de Escenarios en Sistemas de Caficultura Campesina en Puriscal, Costa Rica. Tesis de PhD. San

- José, Costa Rica, Universidad de Costa Rica. 249 pp. 2004. Pérez, HR. El cognitivismo "clásico" o procesamiento de información. Consultado en octubre de 2009. En línea: http://www.robertexto.com/archivo8/cognit_clasico.htm

- $\quad$ Sain, G. Seminario-taller: la adopción de tecnologías: la perspectiva del agricultor y sus implicaciones para la elaboración de políticas. CIMMYT /PROFRIJOL / PASOLAC / ICTA, San José, CR. 350 p. 1999.

- $\quad$ Sinclair, FL; Walker, DH; Joshi, L; Ambrose, B; Tapa, B. Use of a local knowledge based systems approach in the improvement of tree fodder resources on farmland in the eastern hills of Nepal. Bangor, UK, University of Wales. 1993.

- Sinclair FL;Walker DH. 1999. A utilitarian approach to the incorporation of local knowledge in agroforestry research and extension. In Buck, LE; Lassole, JP;

- Fernández, ECM. eds. Agroforestry in sustainable agricultural systems. Estados Unidos, CRC Press. p. 245-275. Strauss, A; Corbin, J. 1990. Basics of qualitative research: grounded theory, procedures and techniques. Newbury Park, US, Sage. 270 p.

1. Profesor Asociado, Grupo Sistemas Agroforestales Pecuarios, Universidad del Tolima, lbagué, Colombia.

2. Investigador, Programa SIRECO, Universidad de Costa Rica.

3. Profesor, Facultad de Ciencias Agroalimentarias, Universidad de Costa Rica.

4. Las "palabras clave" están constituidas por aquellas expresiones que son el eje de un campo nocional. Instaladas en el centro de una red de asociaciones, estas palabras son la "llave" que permite reconstruir una visión del mundo (Guiraud 1962). 